\title{
The Use of HCG-Based Combination Therapy for Recovery of Spermatogenesis after Testosterone Use
}

\author{
Evan P. Wenker, BS, ${ }^{*}$ James M. Dupree, MD, ${ }^{\dagger}$ Gavin M. Langille, MD, ${ }^{\ddagger}$ Jason Kovac, MD, ${ }^{\S}$

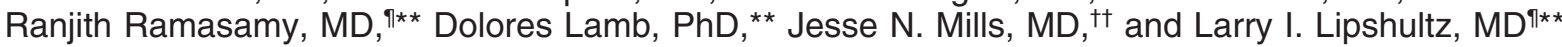 \\ *Baylor College of Medicine, Houston, TX, USA; 'Department of Urology, University of Michigan, Ann Arbor, MI, USA; \\ ¥Department of Urology, Dalhousie University, Saint John, NB, Canada; §Urology of Indiana, Male Reproductive \\ Endocrinology and Surgery, Carmel, IN, USA; "Scott Department of Urology, Baylor College of Medicine, Houston, TX, \\ USA; ${ }^{* \star}$ Center for Reproductive Medicine, Baylor College of Medicine, Houston, TX, USA; ${ }^{\dagger}$ The Urology Center of \\ Colorado, Denver, CO, USA
}

DOI: $10.1111 /$ jsm. 12890

\section{A B S T R A C T}

Introduction and Aim. About 3 million men take testosterone in the United States with many reproductive-age men unaware of the negative impact of testosterone supplementation on fertility. Addressing this population, we provide an early report on the use of human chorionic gonadotropin (HCG)-based combination therapy in the treatment of a series of men with likely testosterone-related azoospermia or severe oligospermia.

Methods. We retrospectively reviewed charts from two tertiary care infertility clinics to identify men presenting with azoospermia or severe oligospermia $(<1$ million sperm $/ \mathrm{mL})$ while taking exogenous testosterone. All were noted to have been placed on combination therapy, which included 3,000 units HCG subcutaneously every other day supplemented with clomiphene citrate, tamoxifen, anastrozole, or recombinant follicle-stimulating hormone (or combination) according to physician preference.

Main Outcome Measure. Clinical outcomes, including hormone values, semen analyses, and clinical pregnancies, were tracked.

Results. Forty-nine men were included in this case series. Return of spermatogenesis for azoospermic men or improved counts for men with severe oligospermia was documented in 47 men (95.9\%), with one additional man (2.1\%) having a documented pregnancy without follow-up semen analysis. The average time to return of spermatogenesis was 4.6 months with a mean first density of 22.6 million $/ \mathrm{mL}$. There was no significant difference in recovery by type of testosterone administered or supplemental therapy. No men stopped HCG or supplemental medications because of adverse events. Conclusions. We here provide an early report of the feasibility of using combination therapy with HCG and supplemental medications in treating men with testosterone-related infertility. Future discussion and studies are needed to further characterize this therapeutic approach and document the presumed improved tolerability and speed of recovery compared with unaided withdrawal of exogenous testosterone. Wenker EP, Dupree JM, Langille GM, Kovac J, Ramasamy R, Lamb D, Mills JN, and Lipshultz LI. The use of HCG-based combination therapy for recovery of spermatogenesis after testosterone use. J Sex Med 2015;12:1334-1337.

Key Words. Human Chorionic Gonadotropin; Testosterone; Azoospermia; Infertility

\section{Introduction and Aims}

$\mathrm{T}$ estosterone supplementation therapy (TST) use among men of reproductive years is

Financial Support: None. becoming increasingly common, with an estimated 3 million men on TST in the United States [1]. Exogenous testosterone use can result in sterility because of suppression of the hypothalamicpituitary-gonadal axis with resulting decreased follicle-stimulating hormone (FSH) and luteinizing 
hormone $(\mathrm{LH})$ release from the anterior pituitary [2]. Low levels of LH lead to decreased intratesticular testosterone production by Leydig cells. This decreased testosterone level, coupled with reduced Sertoli cell function from blunted FSH secretion causes diminished spermatogenesis in the seminiferous tubules [3]. Many men are unaware of this side effect and present for infertility evaluations while on TST [4].

No consensus currently exists on the optimal treatment regimen for men with TST-induced infertility. While previous studies suggested possible irreversible damage to spermatogenesis due to TST, a recent meta-analysis showed no permanent loss of spermatogenesis in men previously on TST [5]. However, recovery may take a year or more and sperm production may not return to pretreatment levels. In addition, sudden withdrawal of TST renders patients symptomatically hypogonadal until endogenous testosterone production returns to normal levels. Ideally, therapy would support return of spermatogenesis quickly with minimal side effects. As such, human chorionic gonadotropin (HCG) is a particularly promising medication because of its ability to raise suppressed serum and intratesticular testosterone levels that are necessary for normal spermatogenesis [6].

Here we present a preliminary, observational report of the use of HCG-based combination therapy in the treatment of men with TST-related infertility.

\section{Methods and Main Outcome Measures}

\section{Patient Selection}

Men presenting for infertility evaluations while on TST from 2006 to 2013 were retrospectively identified from medical records at two andrology clinics in Texas and Colorado. All patients received a complete infertility evaluation by fellowshiptrained andrologists. Patients were included in the analysis if they were using testosterone at initial evaluation and had a semen analysis demonstrating azoospermia or severe oligospermia (density $<1$ million/mL). Men were excluded if they had a history of surgical or congenital sterilization (i.e., vasectomy or congenital absence of the vas deferens), refused to stop TST, or did not have available follow-up semen analyses or pregnancy data after starting HCG. Relevant TST information was extracted from the charts, including modality (injectable, transdermal, pellet, or unknown) and duration of use.

\section{HCG-Based Intervention}

At the initial evaluation, all men stopped TST and began a combination regimen with 3,000 HCG units administered subcutaneously every other day as primary therapy. This dose and interval were chosen to accommodate the half-life of HCG (approximately 33 hours), aiming to provide patients with relatively stable HCG levels. HCG therapy was supplemented with clomiphene citrate, tamoxifen, anastrozole, or recombinant FSH (or a combination) according to physician preference and clinical situation. The supplemental therapies were added to raise native FSH levels, as HCG does not raise FSH levels and has no activity on FSH receptors. Semen and hormone analyses were performed after 4 weeks of HCG therapy and monthly thereafter until semen parameters became stable or a pregnancy was achieved.

\section{End Points}

The primary end point was recovery of any sperm in the ejaculate in men who were azoospermic or an increase in density in those who were severely oligospermic. Achieving a documented pregnancy was a secondary end point. Data were analyzed using Student's $t$-tests and chi-squared tests with a significance value of $P<0.05$. The Baylor College of Medicine Institutional Review Board approved the study.

\section{Results}

Forty-nine men, aged 27 to 63 (mean 40.5, median 40), were identified. Forty-five (91.8\%) were azoospermic at presentation; the remaining four $(8.2 \%)$ were severely oligospermic. These men had been using TST for an average of 52.4 months prior to initiating HCG-based combination therapy. Sixteen $(32.7 \%)$ had used injectable testosterone, $16(32.7 \%)$ had used transdermal testosterone, 7 (14.3\%) used pellets, $6(12.2 \%)$ used some combination of the three, and $4(8.2 \%)$ used unknown types of TST. All were placed on 3,000 units HCG every other day after discontinuing TST. For supplemental medications, 35 (71.4\%) were prescribed clomiphene citrate, 28 $(57.1 \%)$ were prescribed tamoxifen, $10(20.4 \%)$ were prescribed anastrozole, and $1(2.0 \%)$ was prescribed recombinant FSH. The mean duration of HCG-based combination therapy was 14 months. The mean initial FSH, LH, and testosterone levels in subjects prior to stopping TST 
Table 1 Testosterone $(T)$ levels and time to sperm recovery for infertile men $(n=49)$ presenting on various types of testosterone therapy

\begin{tabular}{|c|c|c|c|c|c|c|}
\hline & & $\mathrm{n}$ & Age* (SD) $^{*}$ & $\begin{array}{l}\text { Mean } \\
\text { testosterone } \\
\text { prior to HCG } \\
\text { therapy (SD) }\end{array}$ & $\begin{array}{l}\text { Mean } \\
\text { testosterone }{ }^{\dagger} \\
\text { while on } \mathrm{HCG} \\
\text { therapy }(\mathrm{SD})\end{array}$ & $\begin{array}{l}\text { Mean time (months) } \\
\text { to first sperm } \\
\text { recovery }{ }^{\ddagger} \\
\text { or improvement }^{\S}\end{array}$ \\
\hline \multirow{5}{*}{$\begin{array}{c}\text { Testosterone } \\
\text { therapy }\end{array}$} & Injection & 16 & $41.3(9.0)$ & $610(398)$ & $542(244)$ & $4.4(4.2)$ \\
\hline & Transdermal & 16 & $39.0(6.5)$ & 645 (288) & 447 (137) & $4.4(5.0)$ \\
\hline & Pellet & 7 & $43.9(9.5)$ & 498 (324) & 465 (215) & 3.4 (1.3) \\
\hline & Combination & 6 & $42.8(3.3)$ & 503 (442) & 399 (127) & $6.2(3.4)$ \\
\hline & Unknown & 4 & $33.5(4.7)$ & 378 (177) & 475 (213) & $5.7(3.1)$ \\
\hline ANOva $P$ value & & & 0.25 & 0.66 & 0.63 & 0.78 \\
\hline
\end{tabular}

*In years

†'ln $\mathrm{ng} / \mathrm{dL}$

程or men initially azoospermic

$\S$ For men initially severely oligospermic

ANOVA, analysis of variance; HCG, human chorionic gonadotropin; SD, standard deviation

were $1.39 \mathrm{mIU}, \quad 0.85 \mathrm{mIU}$, and $573.5 \mathrm{ng} / \mathrm{dL}$, respectively. The mean duration of follow-up was 14 months and 686 total months for the entire cohort.

After starting HCG-based therapy, return of spermatogenesis or improvement in sperm density $>1$ million $/ \mathrm{mL}$ was documented in $47(95.9 \%)$ men. One additional man reported a pregnancy but did not have a semen analysis performed. Including this individual, $98.0 \%$ of men were able to recover spermatogenesis. The average time to first sperm recovery in the ejaculate (for azoospermic men) or improvement above 1 million/mL (for severely oligospermic men) was 4.6 months; the mean first sperm density was 22.6 million/mL. At this milestone, the average FSH level was $4.04 \mathrm{mIU}$, an increase of $191 \%$ over the initial value. Similarly, the average LH level was $3.12 \mathrm{mIU}$, an increase of $267 \%$. Average testosterone levels were $475.8 \mathrm{ng} / \mathrm{dL}$ when sperm first returned to the ejaculate, a $17.0 \%$ decrease from levels achieved while on TST $(573.5 \mathrm{ng} / \mathrm{dL}$, $P=0.11)$.

As seen in Table 1, there were no significant differences in mean time to first sperm recovery based on type of TST used (injection, 4.4 months; transdermal, 4.4 months; pellet, 3.4 months; combination, 6.2 months; $P=0.72$ ) or type of supplemental therapy used (clomiphene, 3.2 months; tamoxifen, 4.1 months; anastrozole, 7.5 months; mixed, $5.5 ; P=0.14)$.

Nineteen men (38.8\%) achieved a clinically documented pregnancy during the mean 14 months of follow-up, with no significant difference by type of TST or supplemental therapy used. No men discontinued therapy because of adverse effects.

\section{Discussion}

This early observational report suggests that using HCG-based therapy supplemented with clomiphene citrate, tamoxifen, anastrozole, or a combination can be used to quickly restore spermatogenesis in individuals with azoospermia or severe oligospermia because of previous TST. The return of, or improvements in, spermatogenesis was independent of the type of testosterone used and did not vary significantly based on type of supplemental therapy.

Previous studies have demonstrated that HCG administration can increase intratesticular testosterone levels to normal concentrations, but the drug has not been studied in this current context. ${ }^{6}$ We postulate that supplemental medications are necessary to support FSH production, which cannot be accomplished with HCG alone, and clomiphene citrate and tamoxifen have been shown to increase both gonadotropins [7].

There are currently no studies on the natural history of unassisted sperm recovery after prolonged testosterone use. Some estimates could be derived from the male contraception literature but it is important to consider that patients in the male contraception studies were treated in the closely monitored context of a clinical trial and used testosterone for a maximum of 18 months, making them an unrealistic comparison group. Nevertheless, in an analysis of World Health Organization studies where testosterone was used for 18 months or less to induce contraceptive infertility, recovery of sperm concentration to $>20$ million/mL took an average of 3.2 months [8]. Our study showed similar recovery times in a clinical environment where patients had been using TST for an average 
of 52 months and did so without patients experiencing decreases in testosterone levels.

This HCG-based combination regimen has a number of advantages, including its use of established (albeit off-label), well-tolerated medications. Additionally, this regimen accomplishes the goal of inducing a speedy recovery of spermatogenesis. However, this study's single-arm, observational, and retrospective nature makes definitive proof of this HCG-based combination therapy's efficacy impossible to prove. Future studies should focus on comparing this combination regimen with HCG monotherapy, oral monotherapy, and unaided testosterone cessation, as well as the ability of HCG to mitigate hypogonadal symptoms associated with abrupt discontinuation of TST.

Corresponding Author: Larry I. Lipshultz, MD, Scott Department of Urology, Baylor College of Medicine, 6624 Fannin St., Ste. 1700, Houston, TX 77030, USA. Tel: 713-798-6163; Fax: 713-798-6007; E-mail: larryl@ bcm.edu

Conflict of Interest: The authors report no conflicts of interest.

\section{Statement of Authorship}

Category I

(a) Conception and Design

Evan P. Wenker; James M. Dupree; Gavin M. Langille; Jason Kovac; Ranjith Ramasamy; Dolores Lamb; Jesse N. Mills; Larry I. Lipshultz

(b) Acquisition of Data Evan P. Wenker; James M. Dupree; Gavin M. Langille; Jason Kovac; Ranjith Ramasamy; Dolores Lamb; Jesse N. Mills; Larry I. Lipshultz

(c) Analysis and Interpretation of Data Evan P. Wenker; James M. Dupree; Jesse N. Mills; Larry I. Lipshultz

\section{Category 2}

(a) Drafting the Article

Evan P. Wenker; James M. Dupree; Jesse N. Mills; Larry I. Lipshultz

(b) Revising It for Intellectual Content

Evan P. Wenker; James M. Dupree; Gavin M. Langille; Jason Kovac; Ranjith Ramasamy; Dolores Lamb; Jesse N. Mills; Larry I. Lipshultz

\section{Category 3}

(a) Final Approval of the Completed Article Evan P. Wenker; James M. Dupree; Gavin M. Langille; Jason Kovac; Ranjith Ramasamy; Dolores Lamb; Jesse N. Mills; Larry I. Lipshultz

\section{References}

1 Bagatell CJ, Bremner WJ. Androgens in men-uses and abuses. N Eng J Med 1996;334:707-14.

2 Rahnema CD, Lipshultz LI, Crosnoe LE, Kovac JR, Kim ED. Anabolic steroid-induced hypogonadism: Diagnosis and treatment. Fertil Steril 2014;101:1271-9.

3 Manetti GJ, Honig SC. Update on male hormonal contraception: Is the vasectomy in jeopardy? Int J Impot Res 2010;22:159-70.

4 Coward RM, Rajanahally S, Kovac JR, Smith RP, Pastuszak AW, Lipshultz LI. Anabolic steroid induced hypogonadism in young men. J Urol 2013;190:2200-5.

5 Rastrelli G, Corona G, Mannucci E, Maggi M. Factors affecting spermatogenesis upon gonadotropin-replacement therapy: A meta-analytic study. Andrology 2014;2:794-808.

6 Coviello $\mathrm{AD}$, Matsumoto AM, Bremner WJ, Herbst KL, Amory JK, Anawalt BD, Sutton PR, Wright WW, Brown TR, Yan X, Zirkin BR, Jarow JP. Low-dose human chorionic gonadotropin maintains intratesticular testosterone in normal men with testosterone-induced gonadotropin suppression. J Clin Endocrinol Metab 2005,90:2595-602.

7 Hwang K, Walters RC, Lipshultz LI. Contemporary concepts in the evaluation and management of male infertility. Nat Rev Urol 2011;8:86-94.

8 Ly LP, Liu PY, Handelsman DJ. Rates of suppression and recovery of human sperm output in testosterone-based hormonal contraceptive regimens. Hum Reprod 2005;20:173340 . 